To appear in Practice (in press). Pre-proof copy. Please do not circulate without authors' permission.

\title{
Undertaking family-focused interventions when a parent has a mental illness - possibilities and
}

\author{
challenges
}

\section{Scott Yates $^{1} \&$ Lina Gatsou ${ }^{2}$}

1. Department of Social Work and Youth and Community Development, De Montfort University.syates@dmu.ac.uk (corresponding author)

2. Leicestershire Partnership NHS Trust \& Faculty of Health and Life Sciences, De Montfort University

Paper word count: 6,392 (including abstract and references)

\begin{abstract}
Parental mental illness (PMI) is a public health issue associated with risks of negative outcomes for children and families. Effective whole family interventions with families with PMI are still not well implemented across mental health, social work and multi-agency workforces. This paper presents research with professionals trained in a new programme, the Think Family-Whole Family Programme, to strengthen family-focused work around PMI. It examines professionals' practice and understanding of PMI and identifies enablers of effective practice.
\end{abstract}

Findings indicate that participants identify having appropriate knowledge of how PMI can affect families and the confidence to address it as important factors enabling effective work with them. They also had concerns around how to discuss mental health due to issues of stigma and management support of whole-family work. Results indicate potential for training to achieve positive outcomes in improving professionals' knowledge and confidence and encouraging whole-family work around PMI. Possibilities and challenges for future work with families with PMI are discussed.

\section{Introduction}

Parental mental illness (PMI) is an issue of increasing concern for mental health services, social work and allied professions (Reupert et al, 2011). Although not necessarily a precursor of other problems, $\mathrm{PMI}$ is associated with increased likelihood of caring responsibilities and inconsistent role expectations for children and strain on parent-child relationships (Cooklin, 2013). It can impact the social inclusion, quality of life and emotional and cognitive development of children (Van Loon et al., 2014).

There is growing awareness that to effectively understand and work with PMI, it is important to move beyond individualised, medicalised models of the illness as pathology residing in the ill parent and take account of family and social contexts within which illness manifests and recovery is to be managed (Foster et al, 2012).

PMI is identified as an Adverse Childhood Experience (ACE), associated across populations with poor long-term health and social outcomes (Hughes et al., 2017). The ACE framework, which focuses on the cumulative effect of adverse experiences and associated trauma on long-term outcomes, has been important in showing the widespread distribution of adverse experiences and the social distribution of these conditions as a contributing factor to health inequalities (Kelly-Irving \& Delpierre, 2019). However, it is not without problems. Criticisms focus on its use as a diagnostic 
To appear in Practice (in press). Pre-proof copy. Please do not circulate without authors' permission.

device to "score" individuals in terms of overall "risk" in a way that frames all ACE conditions as inherently negative and can lead to a fatalistic, stigmatising over-biologisation of social experiences (Edwards et al., 2017). It is well-established that the distribution of mental illness follows a socioeconomic gradient, being significantly more common amongst the poorest-off, and is strongly explained in terms of social causation - poverty and adverse social conditions precipitating a worsening of mental health (e.g. WHO, 2007).

Negative conceptualizations of mental illness as innately harmful and the associated stigma is a widespread problem that negatively impacts those affected (Hinshaw, 2005). This can affect not only the ill parent, but also children if they blame themselves for their parent's illness, misinterpret their symptoms as rejection, or become anxious for their parent's safety or their own mental health (Cooklin, 2013). A lack of understanding can also leave ill parents feeling unsupported and instigate negative cycles of stress and conflict (authors, 2015).

Negative effects of stigma are not limited to the parent and their illness, but are associated with role-expectations and self-identity as parents, and these are liable also to impact children through "courtesy stigma" (the extension of the impacts of stigma to people in close relationships with those stigmatized) (Hinshaw, 2005). Social isolation or withdrawal, often a response to stigma, can also affect whole families and reduce help-seeking (Hinshaw, 2005).

There is also growing awareness that family-focused rather than individual interventions can improve recovery, reduce burdens on children and protect children's mental health (Reupert et al, 2011). Current developments include using behavioural family therapy for families with PMI (Pikhala et al., 2011), family-focused psychoeducational interventions (Grove et al, 2015), relational recovery in mental health nursing (Foster \& Isobel, 2017), and interventions to strengthen family communication (Cooklin, 2013; Falkov, 2017).

However, PMI is often hidden, service engagement is low (Moses, 2013), and effective familyfocused practice remains under-developed, especially in social care, education and social work (Ofsted, 2013). There is also a shortage of research examining effectiveness of family-focused interventions in practice settings (Afzelius et al., 2018).

In this paper, we outline development of a family-focused intervention in the UK for use by multiagency professionals, including social workers, in supporting families with PMI. We present evidence from research with professionals involved in this work to consider how it furthers our understanding of the needs of these families and the professionals who work with them, good practice in support and intervention, and barriers to and enablers of positive outcomes.

\section{Outline of the programme}

Although some family-focused work is emerging, there is lack of family-focused mental health training for social work, social care, health and allied services, and there is no structured intervention in the UK to provide family-focused support for families with PMI. In response to this, we were funded by the Department of Health and the NHS Health Innovation and Education Cluster to develop an intervention for use across multi-agency services.

SCIE (2011) stressed the importance of a "no wrong door" principle, meaning families with PMI should not depend on specialist services for support, but whichever services they come into contact with should be equipped to offer support around PMI and where necessary work coherently with more specialised agencies. It was thus important that the programme be relatively easy to understand and apply for a range of services. As families with PMI are known to be a group affected by stigma and with reduced help-seeking, it was also important that the programme be voluntary and flexible. 
To appear in Practice (in press). Pre-proof copy. Please do not circulate without authors' permission.

We identified two existing protocols with evidence of positive outcomes which contained elements of what was required - The Family Model (Falkov, 2017), and The Meriden Family Programme (Fadden, 1998). The Family Model encompasses family-focused communication facilitated between professionals and family members to deepen understanding of one another's experiences, but it is relatively unstructured and focuses on principles for interaction rather than a structured intervention. The Meriden Programme is a practical, skills-based, psycho-educational intervention for supporting families when a young person (18-25) experiences first-onset psychosis. It provides a structured eight-session intervention based around shared mental health literacy, understanding and recognising symptoms, and developing shared staying-well plans. It is, however, designed to work specifically with psychosis, and focuses on families where it is a young person rather than a parent experiencing illness. Adaptation of these programmes was undertaken through stakeholder, public and service-user engagement, with input from clinicians, service managers and executives, commissioners, researchers, service users and young carers.

The final programme - The Think Family-Whole Family Programme (TFWFP) - comprises a two-day training programme for professionals and an eight-session intervention for use with families. The first half of the first day of the training delivers awareness-raising by providing professionals with information on the incidence, context and impacts of PMI. The second half-day introduces The Family Model principles of family-focused communication that contextualise the illness experience in the relational, institutional and social contexts in which families live. The second day outlines the eight-session structure, provides tools for use with families in assessment, communication and goal setting, and uses videos and role-play to familiarise professionals with use of the intervention and The Family Model principles of family communication.

These principles (Falkov, 2017) focus not on individual pathology, but on open, non-judgemental communication around what all family members struggle with, what strengths they have in the contexts of their individual experiences, relationships, services, and the social, community and cultural contexts in which they live. This includes issues of stigma, and relationships outside the family, and acknowledging the role of social stressors connected to poor mental health - financial hardship and precarity, employment, education, housing, etc.). The collaborative work of professionals with families ensures children's voices are heard, builds shared understandings, allows families to make sense of their experiences and to strengthen relationships. Although this perspective focuses on mental illness in families, it does not do so through a medical model lens. Rather than medically-defined illness and predetermined definitions of recovery, the focus is on selfdefined experiences of distress and goals for wellbeing, and on the interpersonal and social contexts in which these are situated.

These principles of family-focused collaborative communication were incorporated into an eightsession structure adapted from The Meriden Programme as follows:

Session 1: Engagement, ground rules and assessment - the professional meets the family, introduces the principles of communication that will shape the intervention, and gives everyone the chance to input into assessment of needs and challenges and setting goals for outcomes.

Session 2: Family meeting - the professional introduces family meetings, in which families themselves, outside of their interaction with professionals, can continue to communicate in a non-judgemental manner, ensuring everyone's voice is heard.

Session 3: Information sharing - professionals facilitate a communicative session in which family members are encouraged to share experiences of what is distressing them, what they are struggling with, and to understand one another's experiences.

Session 4: Problem-solving - a six-step approach to identifying and approaching problems is introduced. Each family member is encouraged to write down a problem or goal they have in 
To appear in Practice (in press). Pre-proof copy. Please do not circulate without authors' permission.

their lives, list possible solutions, discuss these and select a preferred solution and a plan to carry it out.

Sessions 5 \& 6: Staying well plans - family members are asked to consider signs related to relapse (in terms of a family member's mental health and/or general family distress). A card-sort of common issues is provided to prompt discussion, and family members are encouraged to produce their own cards that fit their experience.

The final two sessions are open, focusing on revisiting key issues, evaluating progress and reviewing goals. Professionals are supported through group-based reflective meetings and supervision with a trained family therapy supervisor.

We have presented elsewhere general evidence for the success of TFWFP in supporting families (authors, 2015, 2016). Here, we draw on qualitative evidence from professionals who applied the programme with a family on their caseload to explore how they work with families prior to training, the impacts of the training on practice, and what was most effective in supporting families with PMI.

\section{Method}

Two hundred and three multi-agency professionals completed the training. Professionals were recruited through email contact with multi-agency services and family-support teams in two Local Authorities in the Midlands of England. Participants volunteered after being sent an outline of the programme. This included a request that they had in mind a family on their caseload with whom they could put the training into practice post-training.

One hundred and eighty-nine professionals completed questionnaires, the final item of which requested opt-in to focus groups six to nine months after training. Seventy-six professionals opted in, and at point of follow-up 24 were available for participation.

Eight focus groups, lasting between 32 and 105 minutes were conducted with 24 professionals. They were conversational and lightly structured around questions relating to practice, experiences with $\mathrm{PMI}$, impacts of the training and future practice. Example prompts included:

- How did you work with families with PMI before the training?

- How effective do you think your work was then?

- What were the impacts of PMI in families you worked with?

- $\quad$ Did the training change how you work with these families?

Focus groups were digitally recorded and fully transcribed Thematic analysis was selected as the methods for analysis of the transcripts, as a theoretically-flexible approach suitable for addressing a wide range of exploratory research questions through in-depth analysis of qualitative data (Braun \& Clarke, 2008). The analysis was guided by a realist orientation, holding that the goal of the research is to identify underlying factors in social situations that cause different outcomes. This guided the exploration of the ways that professionals identify the needs of families with PMI, the factors enable or hinder good practice, and the impacts the TFWF training had on professionals and their practice.

This orientation guided the coding of the data. Following close reading of all transcripts to generate familiarity, data were coded by hand to identify themes (common patterns across participants' responses). Emerging themes were read iteratively and checked for consistency against other transcripts, and the analysis was refined and re-read against the entire dataset to ensure the identified themes mapped appropriately to all material in the transcripts. 
To appear in Practice (in press). Pre-proof copy. Please do not circulate without authors' permission.

\section{Results}

A range of findings relate to how PMI affected families and the impacts of the TFWFP intervention on their mental health and wellbeing (see authors, 2016). Here, we focus on issues pertinent to professional practice and development for professionals working with families with PMI. Three key themes are presented - the importance of knowledge about impacts of PMI, the importance of worker confidence, and the importance of understanding family-focused work. Possibilities and challenges for future work are also considered.

The importance of professionals' knowledge about impacts of PMI

Participants universally reported significant improvements in their awareness of PMI and their knowledge of how it can impact all family members. In all focus groups, participants commented that prior to the training, whist they may have been aware that PMI was affecting families' lives, they had not fully appreciated the ways in which it can affect and is affected by the context of relationships within and around the family, especially relationships with younger children. As a result, professionals had previously neglected this in their work. For example, one participant stated that the training

'helped me realise that actually even the little issues you can discuss as a whole family, and... actually it's impacting the whole family.'

Another related at greater length:

'These children were very young, 8 and 7, and I think initially prior to doing this I would have thought oh they're 7 and 8 years old, you know, how on earth do you approach that with a child? But it is important that they do understand because he was saying, "If I'm a good boy mummy won't shout." and he was blaming himself. So it was important that he did have that understanding. He knew mummy took tablets but she took them so she could sleep and that was his understanding and, yes, she was going to sleep sometimes at half past eight at night and leaving him downstairs, but that was what his understanding was. So I've got much more awareness now that, yes they're young but they've still got to have that understanding at a certain level and the parents need to know that the children have got to have that understanding.'

Here, the issue is not that the participant did not realise the parent's illness might affect the children, but that they did not anticipate the specific impacts in respect of the children blaming themselves for their parent's symptoms. Gaining knowledge that this was a potential impact of PMI provided the impetus to overcome their initial reticence in discussing PMI with young children and to begin to work with the family around PMI in ways they had not done previously.

In every focus group, participants related that prior to the training they lacked the knowledge that would have led them to raise the issue of PMI. This was grounded in (as above) lack of knowledge of the ways PMI can impact children, a belief that it was difficult or impossible to work with younger children in relation to PMI, and also in more general lack of insight into how PMI might interact with family relationships. The following exchange is an illustration of this, which also presented in all of the groups:

'Int: If you think back to before the training how were you working with a family? When you went to work with a family and you thought "there is parental mental illness here"?

P1: No, I don't think I really thought about, because you go in and you are running as soon as your feet touch the ground, you don't even really stop to think what have the children been told about... 
To appear in Practice (in press). Pre-proof copy. Please do not circulate without authors' permission.

it's like everyone is suffering but no one has actually stopped to talk and explain and do anything. And I certainly didn't think in that way... having that conversation just didn't happen, "what is it you have got? Do you understand it?" It's asking the question which the training gave you, "what does that look like for you?" Because a lot of our families have anxiety and depression but it looks different for every single one of them. So for me I didn't have that awareness before the training maybe to think about that.'

As these extracts show, lack of knowledge was hampering professionals' prior work, and TFWFP was successful in overcoming this and enabling them to work more effectively with families through their gaining a more in-depth knowledge around the contexts of PMI within family relationships.

The importance of worker confidence and the provision of clear tools

The importance of participants feeling confident in undertaking family-focused work around PMI was also universally discussed - specifically, the importance of them feeling confident that they would be able to work effectively on the issue of parental mental illness if they did raise it with families.

Their concern prior to training was that they 'wouldn't have felt competent' to deal with issues a family discussion of mental illness might raise. They felt they would be (as one participant phrased it) 'opening a can of worms' that they could not effectively deal with, with the fear that they could leave families in a worse situation:

'I suppose for me with my limited experience of this, it was risk management really and concerns about increasing the risks to the family, you know, when people became more unwell the risks increased to the other potential family members... and kind of opening cans of worms but not being able to offer support that was intensive enough to be able to manage that'

Participants in all groups reported having felt prior to training that mental illness was a taboo subject, rooted in stigma, and that it was not their place to raise it. One participant expressed this by rhetorically asking 'who am I to dabble in their mental health?' Another described professionals feeling scared to raise PMI with families:

'the training does fit in very well into what we're doing particularly as a team and it does give you the permission and the confidence I suppose to ask those questions... for some professionals it might be quite scary to actually ask those questions because, as I say, you can put the blinkers on and think well no, I don't want to ask those questions'

Hesitancy in raising PMI as an issue to be explored in professionals' work with families was commonly cited as a weakness in prior practice. Participants also highlighted TFWFP's provision of a structured protocol and tools as important in improving their confidence in raising PMI with families:

'For me it was confidence in something different to go into the house with... the fact that it's quite structured, quite prescriptive in what you're doing, gives you a bit more confidence... It's about having those clear steps and knowing that actually, you know, this week we're looking at staying well plans and... having those clear agenda items.'

There were frequent references to this sense of gaining 'permission' to raise the issue of PMI with families or gaining confidence that this was 'the right thing to do'. This was generated by a sense of normalisation of this kind of intervention through exposure to examples of it in the training and through an awareness that family-focused interventions were advocated by respected individuals and organisations, such as SCIE: 
To appear in Practice (in press). Pre-proof copy. Please do not circulate without authors' permission.

'The Think Family approach is actually on the agenda - we're thinking about it. I think learning that and knowing that these things are going on, that there's a strategy in place and I think all that stuff from the SCIE website and the videos that we watched... that really helped me to feel more confident that actually this is ok to do and I think it carries more weight then.'

The importance of understanding the nature and benefits of a whole-family approach

Although all participants were aware of PMI being an issue for families on their caseload, and although all were in a position to undertake whole-family work, also discussed in every group was their realisation after the training that their prior work was not effectively family-focused.

The training not only oriented practitioners to an awareness of PMI, but also fostered an increased awareness of the importance of family-focused communication and what a family-focused intervention entails:

'The focus [of previous work] was very much on the pathology of the child... We obviously met with families... but there wasn't that sort of connected quality with adult mental health... For me Think Family was very useful in that it made that distinction between working individually and doing family therapy'

This was an issue prevalent in all groups: despite participants' work involving engagement with families, their focus was on a pursuing a relatively narrow set of outcomes relating to the core goals of their service, in this case child behaviour (and in others substance use, youth offending, school engagement, etc.). This was at the expense, participants came to realise, of work that more fully considers the family and social context and how all family members experience and respond to distress and illness.

Whilst some workers like the one above, whose service focused specifically on children's issues (children's physical and mental health, behaviour, or school attendance), came to recognise their work lacked focus on how family context affects children's experiences, others who focused on family outcomes or parental issues (such as Family Support Workers), came to recognise that their work was insufficiently inclusive of children. This is seen in the case of a worker who reflected:

'I thought I was very "children friendly", but I don't think in essence I was really... I would make much more of an effort now to do that with all the families that we work with.'

This comment, typical of workers without a specific background in working with children's issues, indicate that, although there were improvements in how their work included children after training, they were starting from a position that did not adequately engage children. Another participant gave an example form her current work:

'the second youngest in the family who was 12, I would never really have spent that much time with her and it really made her think about, she started talking about what she would be like as a parent. And before I would never have done that, she wouldn't have thought about her mental health and... that would never have happened.'

This raises a potential challenge for family-focused work, as it suggests it might be harder for workers without a child-focused background to feel confident in engaging children in family-focused work around PMI. These findings suggest such workers facing an additional learning need in moving towards family-focused work that engages children.

These difficulties notwithstanding, all participants reported that they changed how they work with families after the training. The most common reported changes were increased attention to families' 
To appear in Practice (in press). Pre-proof copy. Please do not circulate without authors' permission.

experiences of PMI and to distress in the family context, and changes to how they include family members in their work:

I have definitely changed the way I approach when I address parenting... I do sort of gently look into actually we need to look at ourselves as parents and if there are mental health issues... addressing how stress can affect your resilience and how if something isn't addressed through that then it is going to escalate. So it's just raising the awareness. So it definitely has changed my approach.'

In every group there were also comments indicating that increased awareness of the family context led to changes not only in individual practice, but in services' 'working culture,' through such means as changing work hours so that children and parents can be seen together, modifying referral practices, or producing service-wide guidelines for ensuring professionals work with whole families. For example, one participant reported the following regarding a new service-level awareness of the importance understanding PMI in whole-family context:

'The knock-on effect of [working using the TFWFP] is I've actually set up a referral system now where people referring from the team, if they've got a certain diagnosis and they've got young children then they're referring to me and if they've got schizophrenia they get the longer programme... And I now work until 6, half 6, on a Monday so that I can accommodate people who work. And that whole service... has come out of this because it's kick-started me.'

Another example, referencing a more general shift in service-level response was typical of responses across the groups:

'We have actually got facilities now in our team to work with families and we didn't have that at all before, and that's a huge shift. And there's a shift in thinking as well, that we need to be looking at whole families and looking at preventing sort of like further damage really that can be done'

\section{Possibilities and challenges for future implementation}

Despite the positive reflections by participants of the impact of TFWFP on their practice, they also identified challenges to future implementation of whole-family work. The most commonly noted were interconnected issues of time, resources and management support. All focus groups included discussions about management support for intensive family-focused work in general and work around PMI in particular. Particularly problematic was pressure on workers to fulfil services' remits as efficiently as possible with an emphasis on client-throughput that militates against longer-term work with families. Participants were also concerned that the stigma of mental illness and parents' fear of judgement from services required preparatory building of rapport and trust before PMI can be addressed - something they feared they might not be permitted time to undertake in future work. For example:

'Our stumbling block has always been the amount of time we have with families. And when we worked with the family when we were doing the project... in terms of the work we did it did work quite well.... But it was the build-up of a rapport that we felt, that we missed out as a service in terms of how much time we get with them. By the time we perhaps reached a point where we were really getting somewhere, in terms of the amount of time we spent with them we should really be finished.'

These issues highlight the challenges of obtaining support for family-focused intervention from managers who are answerable for services' performance on issue-specific targets (such as substance use, children's behaviour, school attendance, or anti-social behaviour). Whilst such challenges may prove difficult to overcome, participants expressed optimism about maintaining their own improved practice through adapting the sessions and tools of the programme to the client and caseload circumstances they would face in future work, even in cases where time pressures or family circumstances might preclude a full eight-session intervention. For example: 
To appear in Practice (in press). Pre-proof copy. Please do not circulate without authors' permission.

'I don't think there's any hard and fast rules, was there? The training was open for discussion and everything else. It was never said you've got to stick to that and do that and whatever else. If you wanted to tweak it to make it fit with a specific family or an individual then do it'

Others commented that even where their ability to undertake intensive work was restricted, elements of the programme could still be used in isolation or in aggregate to address the needs of different families, and tools could be adapted from the programme to inform future practice:

'I've actually suggested to the team that we use [card-sort symptom assessment tools] on a regular basis to develop more complex care plans... And some of the, you know, even the communication things that we've got, that we can use, there's other groups that people are setting up within the team and I've said "Well you could potentially use some of that in your groups." And you can use bits of it definitely. The problem-solving approach is excellent'

Finally, participants also frequently expressed concern about what one called 'cultural drift' - the return, under pressures on time and throughput, to previous ways of working that neglected the family context of PMI. The provision of ongoing supervision and group discussion went some way to addressing participants' concerns in this regard, and both these elements of the programme also elicited positive comments relating to sustainability.

\section{Discussion}

The findings demonstrate positive potential for multi-agency workforce training based on the principles of family-focused communication and awareness-raising regarding the impacts and contexts of PMI.

One limitation of this study is that, as participation was voluntary, there are potential effects connected to selection bias in participants volunteering for training and focus groups. Additionally, whilst focus groups took place six to nine months after training, we cannot make claims for longerterm impacts and sustainability. Future work should seek to address these issues as well as connect these findings to more rigorous assessments of impacts on families' mental health and wellbeing.

Our findings support those from other studies suggesting that many professionals in contact with families with PMI lack awareness of its impacts and confidence to work with it, and leave it unaddressed in favour of a pre-defined service-specific remit (such as parenting, substance misuse, education or child behaviour) (e.g. Reupert \& Maybery, 2011). Coupled with the reluctance of parents with PMI to engage with social services, these factors see many families who would benefit from family-focused support fall through the net of provision (SCIE, 2011). This research adds to previous work by providing a deeper understanding of the factors underlying services' engagement (or lack of it) with PMI and of how these can be overcome to improve practice.

The work demonstrates that, despite professionals' awareness that PMI affected families they worked with, there was a failure to engage with it in family context. This was due to a range of factors, namely:

In relation to lack of knowledge about impacts of PMI:

- A belief that it would be impossible or inappropriate to engage children in work on PMI.

- A limited understanding of how PMI interacts with families lives and relationships, and especially impacts of children's understanding of their parent's behaviour.

In relation to professionals' lack of confidence in understanding of PMI and their ability to work with it: 
To appear in Practice (in press). Pre-proof copy. Please do not circulate without authors' permission.

- The concern they would not know how to work effectively with families if they began focusing on PMI, and that they might even make families' situations worse.

- Anxiety about raising PMI with families due to stigma, and perceiving that only certain (other) professions have "permission" to discuss it

In relation to a lack of focus on whole-family engagement:

- Children were often excluded and not involved in discussions around mental health.

In relation to challenges to embedding effective whole-family work in the future:

- Workload pressures meaning work focused on services-specific issues, targets and throughput rather than the mental health and wellbeing of the family.

The point regarding stigma and professionals' anxiety is perhaps particularly noteworthy. There is an established body of work on how stigma impacts mental health, social engagement, recovery and help-seeking for ill individuals and their families. This finding, however, shows that professionals' fear of broaching a situation they believe families feel stigmatised by means that we need to think of stigma not only as impacting the seeking of help and support by families, but also as making the offering of help and support by services working with them less likely as well.

Despite the initial barriers to effective work with families around PMI, all participants reported improvements in their practice after training. The factors identified as responsible for these improvements align with the barriers to effective work that were retrospectively identified, and these hold lessons for future work across services. Particularly important is ensuring that professionals have a sound knowledge of mental health and the needs of family members who previously may not have been included in their work - most frequently children, but also partners and other family members. Cultivating an understanding of the nature of whole-family engagement is also important in ensuring that professionals engage all family members. Fostering discussion of the social context of families' experiences and sources of stress that impact mental health was also important.

It was also found that having clear tools and a structured intervention plan improves worker confidence and ameliorates their hesitancy to engage with PMI and to include all family members in support. An important process in tackling professionals' concerns around stigma was the normalisation of whole-family work on PMI through illustrations of such work in practice and familiarisation with official guidelines prescribing it.

Findings also emphasise the importance of obtaining management support for whole-family work, more intensive engagement with families on their own terms, and supervision to ensure maintenance of good practice. Pressure from management relating to client throughput, caseload management and resources was the biggest challenge identified to future work.

\section{Conclusion}

A range of agencies, including (but not limited to) social work, are sites of contact with families with PMI who are often reticent to disclose their illness or to seek help and who may not be in contact with other sources of support. This research shows the potential of a relatively brief training and intervention package based on principles of family communication for improving how services work with these families. It also identifies a number of challenges that work in this area needs to address.

Prior to training, practice in the services that were part of the research saw many families not receiving effective support. A significant problem relates to a form of silo-based services who 
To appear in Practice (in press). Pre-proof copy. Please do not circulate without authors' permission.

emphasise working on a specific targeted problem (such as behaviour or school attendance) in as efficient a manner as possible. This does not allow for exploration of the inter-personal and social contexts surrounding and interacting with these issues and causing distress for families. Participants reported awareness of such issues, of which PMI is a major one, but the pressure to conduct their target-focused work often came at the expense of engaging with the more complex needs and challenges in the lives of the people they work with and their families.

Additionally, participants' accounts indicate that there is not the knowledge base nor the practitioner confidence to work effectively with PMI in any case, and whole-family work is often under-developed, with mental health issues neglected, and children in particular frequently left out of engagement and interventions.

This work demonstrates the possibility for improving skills and practice through a focus on building skills, providing tools to use in interventions, and raising awareness of the ways in which mental illness and distress is situated critically in individuals' and families' interpersonal and social contexts. There is a need for raising awareness of these issues with managers as well as workers to ensure practitioners who work with families are supported in undertaking relevant work. This is something likely to be important in the success of future work and something we have now incorporated into current work using TFWFP.

Here we have discussed impacts of this training on professionals' skills and confidence and on changes in practice. Elsewhere (authors, 2015) we demonstrate also that this has further impacts on families, as improved understanding, communication and goal-setting and interventions with social stressors (such as housing, workplace bullying or school conflict) lead to improvements in family relationships and wellbeing.

\section{Acknowledgement}

This work was supported by Department of Health/NHS Health Innovation and Education Cluster [grant number $x x x]$

\section{Ethical approval}

Ethical approval was given by $x x x$ University Research Ethics Committee

\section{References}

Afzelius, M., Plantin, L. \& Östman, M. (2017) Families living with parental mental illness and their experiences of family interventions. J Psychiatr Ment Health Nurs. 25(2), 69-77

Authors $(2015,2016)$ to be added

Braun, V. \& Clarke, V. (2008) Using thematic analysis in psychology. Qualitative Research in Psychology, 3(2), 77-101

Cooklin, A. (2013) Promoting children's resilience to parental mental illness: engaging the child's thinking. Advances in Psychiatric Treatment, 19 (3) 229-240

Edwards, R., Gillies, V., Lee, E. Macvarish, J., White, S. \& Wastell, D. (2017)The Problem with ACEs. Submission to the House of commons Select Committee Inquiry into the evidence-base for early years intervention (EY10039)

Fadden, G. (1998) 'Family Intervention'. In Brooker, C. \& Repper, J. (Eds). Serious Mental Health Problems in the Community. Balliere-Tindall, London 
To appear in Practice (in press). Pre-proof copy. Please do not circulate without authors' permission.

Falkov, A. (2017) The Family Model. Journal of Parent and Family Mental Health. 2(2). https://escholarship.umassmed.edu/parentandfamily/vol2/iss2/1

Foster, K. \& Isobel, S. (2017) Towards relational recovery: Nurses' practices with consumers and families with dependent children in mental health inpatient units. International Journal of Mental Health Nursing. 27(2), 727-736

Foster, K., O’Brien, L. \& Korhonen, T. (2012) Developing resilient children and families when parents have mental illness: a family-focused approach. International Journal of Mental Health Nursing. 21(1):3-11

Grove, C., Reupert, A. \& Maybery, D. (2015) Gaining knowledge about parental mental illness: how does it empower children? Child and Family Social Work, 20(4), 377-386

Hinshaw, S. (2005) Parental Mental Disorder and Children's Functioning: Silence and Communication, Stigma and Resilience, Journal of Clinical Child \& Adolescent Psychology, 33(2), 400411

Hughes, K., Bellis, M., Hardcastle, K, Sethi, D., Butchart, A., Mikton, C., Jones, L. and Dunne, M. (2017) 'The effect of multiple adverse childhood experiences on health: a systematic review and metaanalysis', Lancet Public Health, 2(8), 356-66

Kelly-Irving, M. \& Delpierre, C. (2019) A Critique of the Adverse Childhood Experiences Framework in Epidemiology and Public Health: Uses and Misuses, Social Policy \& Society, 18(3), 445-456

Moses, T. (2013) Stigma and family. In P. Corrigan (ed.) The Stigma of Disease and Disability. Washington DC: APA

OFSTED (2013) What about the Children? Joint-Working Between Adult and Children's Services When Parents or Carers Have Mental III Health and/or Drug and Alcohol Problems. Available at: www.ofsted.gov.uk/resources/130066

Pikhala, H, Sandlund, M. \& Cederström, A. (2011) Children in Beardslee's family intervention: Relieved by understanding of parental mental illness. International Journal of Social Psychiatry, 58(6), 623-628

Reupert, A., Foster, K., Maybery, D., et al. (2011) "Keeping families and children in mind": an evaluation of a web-based workforce resource. Child \& Family Social Work, 16, 192-200

SCIE (2011) Think Child, Think Parent, Think Family. SCIE, London

Van Loon, L. M. A., et al. (2014) "The relation between parental mental illness and adolescent mental health: The role of family factors." Journal of Child and Family Studies, 23(7): 1201-1214

WHO (2007) Priority Public Health Conditions. WHO 